\title{
PREIMPLANTATION GENETIC TESTING 30 years of preimplantation genetic testing
}

\author{
Alan H Handyside \\ School of Biosciences, University of Kent, Canterbury, UK \\ Correspondence should be addressed to A H Handyside; Email: alan.handyside@outlook.com
}

Reproduction (2020) 160 E1-E3

July 2020 marks the 30th anniversary of the first pregnancies and live births following IVF, embryo biopsy and single cell genetic analysis in couples at risk of X-linked diseases (Handyside et al. 1990). Since then, preimplantation genetic diagnosis (PGD), or preimplantation genetic testing for monogenic disease (PGT-M), as it has recently been renamed, has become well established as a valuable alternative to prenatal diagnosis for a wide range of inherited conditions and extended to chromosome abnormalities including aneuploidy and structural rearrangements (Handyside 2018, Niederberger et al. 2018, Cimadomo et al. 2020). I am honoured to have been invited to be the Guest Editor of this special issue of Reproduction to highlight this anniversary. The five reviews by leading researchers and clinicians in different aspects of preimplantation genetics and early non-invasive prenatal diagnosis, as well as reflecting on progress over the last 30 years, provide a contemporary perspective on current clinical practice and a glimpse into future developments (Video 1).

\section{Video 1}

Professor Alan Handyside looks back at his work developing the first genetic testing of pre-implantation embryos. It marks the 30th anniversary of his Nature paper, which reported the first pregnancies involving genetically tested embryos. Reproduction has published a special issue marking this anniversary, that contains a collection of reviews presenting a historical perspective, as well as presenting recent and state-of-the-art developments in this exciting field. This video (http:// movie-usa. glencoesoftware.com/video/10.1530/REP20-0412/ video-1) is available from the online version of the article at https://doi.org/10.1530/REP-20-0412.

In the early days, embryos could only be cultured for 2 or 3 days post insemination (day 2 or 3 ) before they were transferred to the uterus, and even delaying transfer to day 4 reduced implantation and pregnancy rates. The slow freezing protocols used for cryopreservation of embryos at these early stages were rudimentary and often embryos were destroyed or partially damaged in the thawing process. The challenge, therefore, was to remove a single cell from each embryo early on day 3 at the 6 - to 10-cell stage to minimise the damage to the embryo, perform the genetic analysis for the mutation on single copies of the parents' chromosomes within a few hours, and then select unaffected embryos for transfer later the same day. This could only be contemplated because of the emergence in the mid-1980s of the PCR, which allowed the million-fold amplification of short fragments of DNA. In addition, human genetics was still in its infancy and the mutations causing only a handful of common inherited diseases had been identified.

Today, there have been major advances in all aspects of preimplantation genetics that have revolutionised our ability to interrogate the genetics of human gametes and embryos. Embryo culture to the blastocyst stage on days 5-7 and biopsy of multiple trophectoderm cells using laser assisted hatching and excision are now routine. Cryopreservation of biopsied blastocysts, using ultrarapid freezing or vitrification, is highly efficient and minimally damaging, removing any time constraints on the genetic analysis. Most importantly, the ability to amplify the whole genome of single or small numbers of cells efficiently and generate micrograms of DNA have revolutionised our ability to perform genetic tests. This has enabled the use of genome-wide methods including microarrays and next generation sequencing (NGS) to genotype SNP markers to provide universal linkagebased tests for any monogenic disease combined with molecular cytogenetic analysis of chromosome abnormalities (Tsuiko et al. 2020). In addition, the development of 'multi-omics' methods, for example, combining genomics and RNA sequencing at the single cell level promise to revolutionise our knowledge of early human development.

Alongside these developments, the sequencing of the human genome in the early 2000s and progress in identifying the genetic basis of thousands of monogenic diseases and other conditions have expanded the range of applications for PGT. These include rare severe 
childhood lethal diseases, late onset diseases, genetic predisposition to cancers and tissue typing to enable cord blood stem cells to be used for transplantation to affected children in the family (De Ryke et al. 2020). Furthermore, pregnancy and live birth rates have significantly improved and the evidence to date suggests that the health of the children born following PGT is not adversely affected at birth or in early childhood.

From the beginning, PGT raised fears of 'designer babies' in which parents would use the technology to select desirable characteristics, such as hair or eye colour, or to prevent the inheritance of less serious conditions. In my experience, beyond some couples wanting to select for gender, there has been no evidence that this is the case and I have long argued that parents can be relied on to make their own informed decisions (Handyside 2010). The use of universal genomewide, linkage-based PGT methods now enable testing for two monogenic diseases, in rare families with a history of both conditions. But beyond this example, the prospect of testing for multiple genetic variants to select for complex traits or to reduce the risk of common diseases was considered to be impractical because of the limited number of embryos available. In recent years, however, population scale whole genome sequencing and genome-wide association studies, such as the 1000 genomes project (Siva 2008), have enabled increasingly accurate polygenic risk scoring (PRS) for a range of common, medically significant conditions, such as cardiovascular disease and diabetes. Based on this information, comprehensive PGT for monogenic disease and chromosome abnormalities can now be extended to polygenic conditions (PGT-P) and provides a PRS for each embryo for multiple common diseases (Treff et al. 2020). This development is controversial and for some raises ethical and societal issues. However, for families with a history of these conditions, there is increasing evidence that PGT-P can identify embryos at high risk.

Chromosome aneuploidy is a major cause of pregnancy failure and loss, and rarely viable abnormal pregnancy and live birth, following both natural conception and IVF. The use of various molecular cytogenetic methods for PGT of aneuploidy (PGT-A) has confirmed the high incidence of aneuploidy in human gametes and embryos, particularly in oocytes in which the incidence increases exponentially in women above the age of 35 years (Rubio et al. 2020). PGT-A following blastocyst biopsy is therefore widely used for euploid embryo selection for infertile patients and there is accumulating evidence for significantly improved implantation rates and live birth outcomes, particularly following single vitrified-warmed embryo transfer in later managed cycles. The use of quantitative, mainly NGS-based methods, for 24 chromosome copy number analysis has revealed a relatively high incidence of intermediate copy number changes interpreted as indicating mosaicism for aneuploidies among the sampled trophectoderm cells. The clinical significance of low-level mosaicism is not fully understood since there are now reports of apparently healthy live births following transfer of embryos with only this type of copy number change. Several years ago, it was discovered that there is cell-free DNA of embryo origin in the blastocoel fluid and the culture medium of embryos cultured to the blastocyst stage. This raises the prospect of non-invasive PGT-A (niPGT-A) which would avoid the need for embryo biopsy and facilitate more widespread application for embryo selection in IVF to improve live birth rates per embryo transferred.

Inherited diseases typically affect all the cells and tissues of the body and the clinical manifestations are often multisystemic. This makes them challenging to treat. However, there has been some progress. For example, there is now a drug which can effectively arrest the neurological degeneration in children affected by Battens disease (Kohlschütter et al. 2019) and there has been some success with gene therapy for beta thalassaemia (Ikawa et al. 2019). These therapies remain highly expensive and limited in their availability. For couples that know that they are at risk of having affected children either because of a clinical diagnosis in an existing child or family member or increasingly because of the availability of preconception screening for extensive panels of serious recessive monogenic diseases, PGT or prenatal diagnosis following natural conception remain the only options for them to have their own healthy children.

Thirty years ago, it was anticipated that early noninvasive methods of diagnosing inherited disease from trophoblast cells recovered from the lower pole of the uterus or from cell-free foetal DNA in maternal blood would be developed and would, within a few years, replace PGT because of the expense and difficulty of the IVF process especially for fertile couples. In the event, it would be over two decades later following the development of NGS that non-invasive prenatal testing (NIPT) for common aneuploidies became possible and only now that this approach is being extended to genome-wide aneuploidies, microdeletion syndromes and some common monogenic diseases (Shaw et al. 2020).

Preimplantation genetic testing remains technically challenging and demanding and requires a multidisciplinary team, including expert genetic counselling to ensure the couple are fully aware of their options and the possible outcomes. Nevertheless, the birth of thousands of unaffected children world-wide is a testament to the value couples place on starting a pregnancy knowing that it is unaffected and a reward for all the dedicated work of those involved in helping them to have healthy children. 


\section{Declaration of interest}

The authors declare that there is no conflict of interest that could be perceived as prejudicing the impartiality of this editorial.

\section{Funding}

This work did not receive any specific grant from any funding agency in the public, commercial, or not-for-profit sector.

\section{References}

Cimadomo D, Rienzi L, Capalbo A, Rubio C, Innocenti F, GarcíaPascual CM, Ubaldi FM \& Handyside A 2020 The dawn of the future: 30 years from the first biopsy of a human embryo. The detailed history of an ongoing revolution. Human Reproduction Update 26 453-473. (https:// doi.org/10.1093/humupd/dmaa019)

De Rycke M, Berckmoes V, De Vos A, Van De Voorde S, Verdyck P, Verpoest W \& Keymolen K 2020 Clinical experience of preimplantation genetic testing. Reproduction 160 A45-A58. (https://doi.org/REP-200082)

Handyside A 2010 Let parents decide. Nature 464 978-979. (https://doi. org/10.1038/464978a)

Handyside AH 2018 'Designer babies' almost thirty years on. Reproduction 156 F75-F79. (https://doi.org/10.1530/REP-18-0157)

Handyside AH, Kontogianni EH, Hardy K \& Winston RML 1990 Pregnancies from biopsied human preimplantation embryos sexed by Y-specific DNA amplification. Nature 344 768-770. (https://doi.org/10.1038/344768a0)

Ikawa Y, Miccio A, Magrin E, Kwiatkowski JL, Rivella S \& Cavazzana M 2019 Gene therapy of hemoglobinopathies: progress and future challenges. Human Molecular Genetics 28 R24-R30. (https://doi. org/10.1093/hmg/ddz172)

Kohlschütter A, Schulz A, Bartsch U \& Storch S 2019 Current and emerging treatment strategies for neuronal ceroid lipofuscinoses. CNS Drugs 33 315-325. (https://doi.org/10.1007/s40263-019-00620-8)

Niederberger C, Pellicer A, Cohen J, Gardner DK, Palermo GD, O'Neill CL, Chow S, Rosenwaks Z, Cobo A, Swain JE et al. 2018 Forty years of IVF. Fertility and Sterility 110 185.e5-324.e5. (https://doi.org/10.1016/j.fertn stert.2018.06.005)

Rubio C, Rodrigo L \& Simón C 2020 Chromosome abnormalities in human embryos. Reproduction 160 A33-A44. (https://doi.org/10.1530/ REP-20-0022)

Siva N 20081000 genomes project. Nature Biotechnology 26 256. (https:// doi.org/10.1038/nbt0308-256b)

Shaw J, Scotchman E, Chandler N \& Chitty L 2020 Non-invasive prenatal testing for aneuploidy, copy number variants and single gene disorders. Reproduction 160 A1-A11. (https://doi.org/10.1530/REP-19-0591)

Treff N, Marin D, Lello L, Hsu S \& Tellier L 2020 Preimplantation Genetic Testing for Polygenic Disease Risk. Reproduction 160 A13-A17. (https:// doi.org/10.1530/REP-20-0071)

Tsuiko O, Fernandez Gallardo E, Voet T \& Vermeesch J 2020 Single-cell technologies at the forefront of PGT and embryo research. Reproduction 160 A19-A31. (https://doi.org/10.1530/REP-20-0102)

Received 24 July 2020

First decision 17 August 2020

Revised Manuscript received 18 August 2020

Accepted 20 August 2020 